\begin{tabular}{|c|l|}
\hline Title & $\begin{array}{l}\text { Structure and Reactivity of A lkoxycarbonyl (Ester)-Terminated Monolayers on Silicon : Sum Frequency Generation } \\
\text { Spectroscopy }\end{array}$ \\
\hline Author(s) & A sanuma, Hidehiko; Noguchi, Hidenori; Uosaki, Kohei; Y u, HuaZ Zhong \\
\hline Citation & $\begin{array}{l}\text { Journal of Physical Chemistry B, 110(10), 4892-4899 } \\
\text { https:/doi.org/10.1021/jp056131+ }\end{array}$ \\
\hline Issue Date & 2006-03-16 \\
\hline Doc URL & http://hdl.handle.net/2115/50223 \\
\hline Type & article \\
\hline File Information & JPCB110-10_4892-4899.pdf \\
\hline
\end{tabular}

Instructions for use 


\title{
Structure and Reactivity of Alkoxycarbonyl (Ester)-Terminated Monolayers on Silicon: Sum Frequency Generation Spectroscopy
}

\author{
Hidehiko Asanuma, ${ }^{\dagger}$ Hidenori Noguchi,, Kohei Uosaki, ${ }^{*}, *$ and Hua-Zhong Yu ${ }^{*, \dagger}$ \\ Department of Chemistry, Simon Fraser University, Burnaby, British Columbia V5A 1S6, Canada, and \\ Division of Chemistry, Graduate School of Science, Hokkaido University, Sapporo 060-0180, Japan
}

Received: October 25, 2005; In Final Form: January 13, 2006

\begin{abstract}
Carboxylic acid-terminated monolayers on crystalline silicon surfaces can be readily modified with biological macromolecules for the fabrication of semiconductor-based biosensing devices. They were prepared by acidcatalyzed hydrolysis of alkoxycarbonyl (ester)-terminated monolayers and studied by vibrational sum frequency generation (SFG) spectroscopy. The $\mathrm{C}-\mathrm{H}$ vibration region of the SFG spectra consists of strong methyl bands with significant contributions from methylene stretching modes, indicating that these monolayers are generally ordered but with considerable gauche defects in the alkyl chains in comparison with $n$-alkyl monolayers. After hydrolysis, the methylene stretching modes prevail, with "residues" of the methyl bands, indicating incomplete hydrolysis and disruption of the monolayer structure. This work demonstrates that SFG is capable of providing quantitative information on structure-reactivity correlations in organic monolayers.
\end{abstract}

\section{Introduction}

Silicon is one of the most widely used semiconductors in modern technology; therefore, an understanding of the fundamental principles underlying its surface chemistry is essential. ${ }^{1}$ Organic monolayers covalently bonded to the silicon surface have gained much attention since their introduction about a decade ago, ${ }^{2}$ because of their well-defined structure and the possibility to introduce diverse electrical and optical functionalities to the system. ${ }^{3}$ The preparation of these monolayers is not sophiscated; they can be readily prepared from hydrogenterminated silicon $(\mathrm{H}-\mathrm{Si})$ and diacyl peroxides, terminal olefins, or Grignard reagents. ${ }^{2-7}$ Electrochemical reduction of aryldiazonium ions on silicon electrodes ${ }^{8}$ and the reaction of alkylmagnesium or alkyllithium reagents with halogenated silicon surfaces ${ }^{9}$ have also been explored as alternative synthetic routes.

$\omega$-Functionalized organic monolayers on silicon allow the immobilization of biomolecules and may therefore be used as platforms for the fabrication of biochips. ${ }^{10-14}$ The ease of further derivatization makes carboxy $(-\mathrm{COOH})$ one of the most versatile functional groups for surface modification. ${ }^{5 \mathrm{a}, 6 \mathrm{~b}, 15}$ Because of the reactivity of carboxylic acids toward hydrogenterminated silicon, ${ }^{5 a}, 16$ carboxy-terminated monolayers are most frequently prepared by acid- or base-catalyzed ester hydrolysis. ${ }^{15}$ In the past, such surface hydrolysis reactions have been mainly investigated by attenuated total reflectance (ATR)-Fourier transform infrared (FTIR) spectroscopy, ellipsometry, and wetting measurements. ${ }^{5 \mathrm{a}, 6 \mathrm{~b}, 15}$ Unfortunately, these techniques provide limited information on the extent of ester hydrolysis and the conformational change of the monolayer structure. In the present study, we have explored the feasibility of using sum frequency generation (SFG) spectroscopy, a novel and powerful spectroscopic technique, to elucidate these fundamental issues, which are critical for proficient immobilization of biological macromolecules.

\footnotetext{
* To whom correspondence should be addressed. E-mail: uosaki@ pcl.sci.hokudai.ac.jp (K.U.); hzyu@sfu.ca (H.-Z.Y.).

Simon Fraser University.

$\doteqdot$ Hokkaido University.
}

SFG spectroscopy is based on a second-order nonlinear optical effect of a photon generated at a frequency equal to the sum of the frequencies of two incident light beams. ${ }^{17}$ It is not observed in media exhibiting inversion symmetry under the electric dipole approximation. Infrared-visible SFG has been utilized most commonly, as it provides information on molecular interactions and orientations. ${ }^{17 \mathrm{~b}, \mathrm{c}}$ Unparalleled surface sensitivity and the ability to detect specific vibrations at buried interfaces with short-time-scale resolution are its main advantages over conventional infrared (FTIR and Raman) techniques. Another unique feature of SFG is its selection rule: to be detectable, the vibrational modes must be both Raman- and IR-active. Furthermore, the polarization combination of SFG, visible, and IR beams determines the SFG intensity observed. Particularly, the ssp (s-polarized SFG, s-polarized visible, and p-polarized IR) combination probes surface vibrational modes with a dipole moment perpendicular to the interface, while the sps and pss combinations detect vibrational modes with a dipole moment parallel to the surface. The ppp combination has no vanishing components; thus, it depends on all tensor elements; that is, ppp-polarized SFG spectra exhibit vibrational modes with both perpendicular and parallel components. All of these features make SFG spectroscopy a highly sensitive, surface-specific technique for studying molecular conformations. ${ }^{18-24}$ Shen and co-workers pioneered SFG studies of molecular interfaces, initially by examining monolayers of coumarin 504 molecules spin-coated on quartz ${ }^{18 a}$ and later by monitoring the $\mathrm{C}-\mathrm{H}$ stretching modes of methanol and pentadecanoic acid adsorbed on glass and water. ${ }^{18 \mathrm{~b}}$ The polarization characteristics of the SFG signals revealed the molecular orientation changes in a Langmuir film of pentadecanoic acids when the surface density was varied. ${ }^{20 \mathrm{c}}$ At the methanol vapor/liquid interface, the terminal methyl groups were found to point away from the liquid with a very broad orientational distribution. ${ }^{18 \mathrm{~d}}$

The study of octadecyltrichlorosilane (OTS) monolayers on silica by Guyot-Sionnest et al. revealed that the alkyl chains were oriented normal to the surface. ${ }^{19}$ Liu et al. examined the adsorption of OTS at silica/solvent interfaces, particularly the 
role of water in the cross-linking chemistry. ${ }^{20} \mathrm{Ye}$ et al. investigated the interactions between water and OTS-modified quartz and found that water molecules flipped at the quartz/ OTS interface, while those at the OTS surface maintained their orientation upon changing the $\mathrm{pH}$ from neutral to acidic. ${ }^{21} \mathrm{Chen}$ et al. detected different molecular structures at polymer/silane interfaces; that is, the silane molecules adopted different conformations at the interface depending on the surface structure of the polymer. ${ }^{22}$ Recently, Voges et al. characterized $\omega$-estersiloxane monolayers on glass before and after hydrolysis; ${ }^{23}$ they observed significant differences between their spectra and those of silica/glass substrates modified with simple long-chain alkyl molecules. ${ }^{24}$ All of these previous studies demonstrate the capabilities of SFG spectroscopy to gain a better understanding of surface chemistry at the molecular level.

SFG investigations of organic monolayers directly bonded to silicon surfaces are limited to date..$^{25-27} \mathrm{Ye}$ et al. have investigated the stability of hydrogen-terminated $\mathrm{Si}(111)$ surfaces by monitoring the $\mathrm{Si}-\mathrm{H}$ stretching mode and reported wavelength-dependent photo-oxidation processes involving adsorbed water molecules. ${ }^{25}$ Ishibashi et al. communicated an SFG study of $n$-alkyl monolayers on $\operatorname{Si}(111)$ surfaces two years ago and suggested that the molecular conformations are chain-length dependent. ${ }^{26} \mathrm{We}$ have recently confirmed the epitaxial arrangement and high conformational order of organic monolayers on silicon. ${ }^{27}$ In particular, we have found that the methylene groups of octadecyl monolayers on $\mathrm{Si}(111)$ are in the all-trans conformation and the tilt angle of the methyl group is about $85^{\circ} .{ }^{27}$ Now, we are extending the scope of our research to the characterization of $\omega$-functionalized organic monolayers on silicon from both the structure and reactivity perspectives.

The following features make SFG spectroscopy particularly suitable for this investigation: ${ }^{17}$ (1) SFG is sensitive to noncentrosymmetric structures such as methyl groups. The progress of ester hydrolysis on the surface should be easily revealed by the disappearance of the methyl $\mathrm{C}-\mathrm{H}$ bands; ATR-FTIR is not sensitive for this task because of the weak IR signals from methyl stretches. (2) SFG can provide information about the order and molecular orientation in the monolayers, particularly the possible structural disturbances caused by hydrolysis. We believe that this SFG study could end the controversy involving the interpretation of the hydrolysis products on silicon surfaces $^{5 \mathrm{a}, 6 \mathrm{~b}, 15}$ and contribute to a better understanding of the structure-reactivity correlation in molecular films.

\section{Experimental Section}

2.1. Materials. All chemicals were of reagent-grade quality and used as received, unless otherwise stated. Milli-Q water $(>18.3 \Omega \cdot \mathrm{cm})$ was used throughout the experiments. 1-Dodecene (98\%), 10-undecylenic acid (98\%), and 1,1,1-trichloroethane (99.5\%) were purchased from Aldrich; tetrahydrofuran (THF), methanol (99.0\%), ethanol (99.0\%), propanol (99.0\%), sulfuric acid $(96 \%)$, and hydrogen peroxide $(30 \%)$ were purchased from Wako Chemicals; and ammonium fluoride (40\%) was purchased from Morita Chemical Industries.

2.2. Synthesis. The long-chain ester molecules, methyl 10undecenoate (Me-UD), ethyl 10-undecenoate (Et-UD), and propyl 10-undecenoate (Pr-UD), were prepared according to the method of Sieval et al. ${ }^{5 a}$ In brief, a mixture of 10 -undecylenic acid $(45.6 \mathrm{~g})$ and the desired alcohol $(50 \mathrm{~mL})$ was allowed to reflux for $3 \mathrm{~h}$ with a few drops of sulfuric acid. For the preparation of Pr-UD, the reaction was carried out in a DeanStark setup and $40 \mathrm{~mL}$ of toluene was added. After removal of alcohols and solvents via vacuum distillation, the crude product was dissolved in diethyl ether. It was then washed with saturated sodium bicarbonate solution, water, and potassium chloride solution and dried over magnesium sulfate. The product was obtained upon vacuum distillation, and its structure was confirmed by ${ }^{1} \mathrm{H}$ NMR spectroscopy. ${ }^{5 \mathrm{a}}$

1-Dodecene was distilled from sodium under reduced pressure (20-30 Torr); Me-UD, Et-UD, and Pr-UD were further purified by passing them through an activated $\mathrm{Al}_{2} \mathrm{O}_{3}$ column.

2.3. Sample Preparation. Silicon (111) wafers (3.0-5.0 $\Omega$ • $\mathrm{cm}$, n-type, donated by Shin-Etsu Semiconductors) were cut into pieces $\left(1.5 \times 2 \mathrm{~cm}^{2}\right)$ and cleaned in "piranha" solution $(3: 1$ mixture of concentrated $\mathrm{H}_{2} \mathrm{SO}_{4}$ and $30 \% \mathrm{H}_{2} \mathrm{O}_{2}$ ) at $90{ }^{\circ} \mathrm{C}$ for 30 min. CAUTION: Piranha solution reacts violently with organic materials; it must be handled with extreme care. After copious rinsing with deionized water, the wafers were etched in deoxygenated $\mathrm{NH}_{4} \mathrm{~F}$ (40\% aqueous solution) to remove the native oxide and obtain hydrogen-terminated silicon $(\mathrm{H}-\mathrm{Si})$. Attenuated total reflectance (ATR) silicon crystals $(25 \times 5 \times$ $1 \mathrm{~mm}^{3}$, Harrick Scientific Inc.) for FTIR measurements were cleaned by the standard RCA procedure, ${ }^{28}$ prior to the etching steps outlined above.

The fresh $\mathrm{H}-\mathrm{Si}$ samples were transferred under argon into Schlenk tubes containing $2-3 \mathrm{~mL}$ of deoxygenated modification reagents and heated to $160{ }^{\circ} \mathrm{C}$ for $4 \mathrm{~h}$. The modified silicon samples were then rinsed at room temperature with THF and 1,1,1-trichloroethane before characterization. The hydrolysis of ester-terminated monolayers on silicon was carried out by immersion in $2.0 \mathrm{M} \mathrm{HCl}$ at $70{ }^{\circ} \mathrm{C}$ for $2 \mathrm{~h}$.

2.4. Surface Characterization. The SFG system employed a picosecond Nd:YAG laser (PL2143B, Ekspla) to pump an optical parametric generation/optical parametric amplification/ difference frequency generation (OPG/OPA/DFG) system, which generates tunable infrared radiation in the range 2.3$8.5 \mu \mathrm{m} .{ }^{27}$ The second harmonic output of the YAG (yttriumaluminum-garnet) laser (532 nm) was used as the visible light. The two beams were loosely focused onto the sample located on a rotational stage, at an incident angle of $70^{\circ}$ for visible light and an incident angle of $50^{\circ}$ for infrared light. Thus, the SFG beam produced was filtered through irises and a monochromator (Oriel Instruments, MS257) and detected by a photomultiplier tube (PMT, Hamamatsu, R3896). The SFG signal is normalized to the intensities of visible and infrared light. The SFG, visible, and IR beams were all p-polarized (abbreviated as ppp). All measurements were carried out in air at room temperature $\left(22 \pm 2{ }^{\circ} \mathrm{C}\right)$.

The following equations are utilized to express the SFG intensity $\left(I_{\mathrm{SFG}}\right)$ and to fit the spectra:

$$
\begin{aligned}
I_{\mathrm{SFG}} & =\frac{8 \pi^{3} \omega_{\mathrm{SFG}}{ }^{2} \sec ^{2} \theta_{\mathrm{SFG}}}{c^{3}}\left|\chi_{\mathrm{eff}}^{(2)}\right|^{2} I_{\mathrm{Vis}} I_{\mathrm{IR}} \\
\chi_{\mathrm{eff}}^{(2)} & =\left|\chi_{\mathrm{NR}}^{(2)}\right| e^{i \phi_{n}}+\sum \frac{A_{n}}{\omega_{\mathrm{IR}}-\omega_{n}+i \Gamma_{n}}
\end{aligned}
$$

where $\chi_{\mathrm{eff}}^{(2)}$ and $\chi_{\mathrm{NR}}^{(2)}$ are the effective second-order nonlinear susceptibilities of the resonant and nonresonant components, respectively; $\phi_{n}$ is the phase angle between the resonant and nonresonant components; and $c, A_{n}$, and $\Gamma_{n}$ are the speed of light in a vacuum, the amplitude, and the homogeneous width of the surface vibration mode $(n)$ corresponding to frequency $\omega_{n}$, respectively.

ATR-FTIR spectra were recorded on a Nicolet Nexus-IR 560 spectrometer equipped with a mercury cadmium telluride (MCT) detector cooled with liquid nitrogen. The ATR crystals 

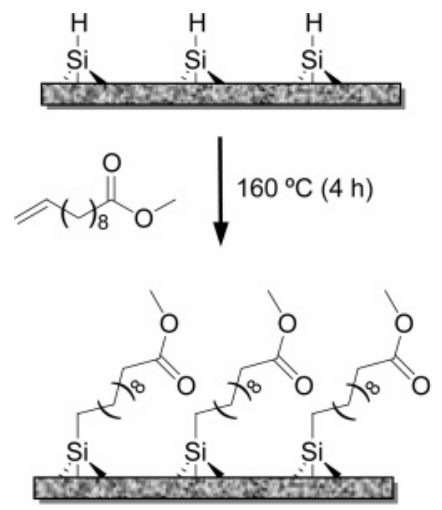

$2.0 \mathrm{M} \mathrm{HCl}$

$70^{\circ} \mathrm{C}(2 \mathrm{~h})$

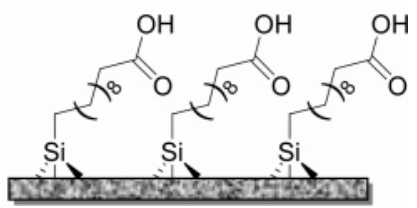

Figure 1. Schematic illustration of the preparation of ester-terminated monolayers on silicon and the subsequent hydrolysis reaction. A methyl 10-undecenoate (Me-UD) monolayer is shown as an example.

TABLE 1: Water Contact Angles on a Silicon Surface Modified with Ester-Terminated Monolayers before and after Hydrolysis

\begin{tabular}{ccc}
\hline monolayer & before hydrolysis $(\mathrm{deg})$ & after hydrolysis $(\mathrm{deg})$ \\
\hline Me-UD & $67 \pm 1$ & $52 \pm 2$ \\
Et-UD & $74 \pm 2$ & $53 \pm 2$ \\
Pr-UD & $77 \pm 1$ & $57 \pm 1$
\end{tabular}

were mounted in a dry air-purged sample chamber with the IR beam focused normal to one of the $45^{\circ}$ bevels. The spectra were collected for 1000 scans at a $2.0 \mathrm{~cm}^{-1}$ resolution, and the background files were obtained with freshly prepared oxidized silicon surfaces. All of the spectra were measured in the 4000$1500 \mathrm{~cm}^{-1}$ range that is limited by the high absorbance of silicon ATR crystals below $1500 \mathrm{~cm}^{-1}$. No corrections were made for either water vapor or atmospheric $\mathrm{CO}_{2}$. In most cases, a linear baseline correction was carefully applied.

Wetting measurements were performed on an AST Optima contact angle system under ambient conditions $\left(18-22{ }^{\circ} \mathrm{C}, 30-\right.$ $35 \%$ relative humidity) using a horizontal light beam to illuminate the water droplet. Contact angles were measured for at least three independent samples $(4-5$ readings at different spots per sample) with $2.0-\mu \mathrm{L}$ drops of deionized water.

\section{Results and Discussion}

Wetting measurements showed that the water contact angles of the three ester-terminated monolayers (Table 1) rise in the following order: Me-UD < Et-UD < Pr-UD monolayer; that is, the hydrophobicity increases with the chain length of the alkoxy group. Upon treatment with dilute hydrochloric acid (2.0 $\mathrm{M} \mathrm{HCl}$ ) for $2 \mathrm{~h}$ at $70{ }^{\circ} \mathrm{C}$, the water contact angles dropped significantly. This increase in hydrophilicity is attributed to the formation of carboxylic acid groups upon hydrolysis (Figure $1)$. However, the values obtained for the hydrolyzed surfaces (in the range $50-57^{\circ}$ ) were substantially higher than those of carboxylic acid-terminated monolayers on gold prepared by direct adsorption of $\mathrm{HS}\left(\mathrm{CH}_{2}\right)_{n} \mathrm{COOH}$, which are essentially zero. $^{29}$ The relatively low hydrophilicity of the carboxylic acid-
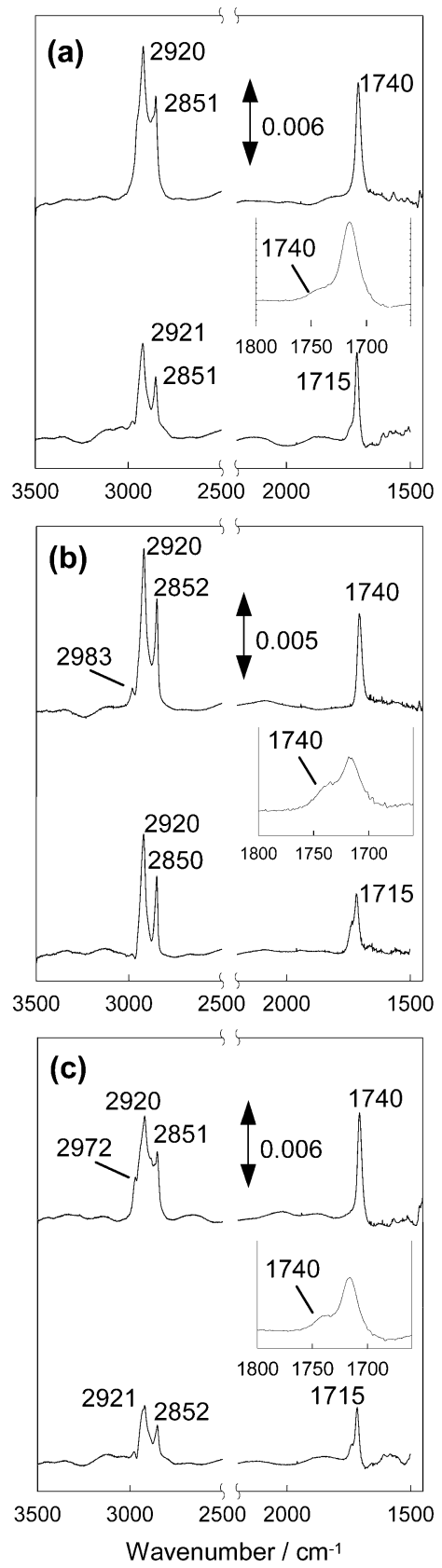

Figure 2. ATR-FTIR spectra of the ester-terminated monolayers on silicon: (a) Me-UD; (b) Et-UD; (c) Pr-UD. In each panel, the top trace and the bottom trace show the spectrum obtained from the monolayer before and after hydrolysis, respectively; the inset shows the enlarged carbonyl region $\left(1650-1800 \mathrm{~cm}^{-1}\right)$.

terminated monolayers on silicon prepared via ester hydrolysis may be attributed to the presence of unreacted ester groups. However, this conclusion is not obvious, as the density of alkanethiolate monolayers on gold is significantly higher than that of alkyl monolayers on $\operatorname{Si}(111),{ }^{2 b}$ which makes a direct comparison difficult. Therefore, the changes of wetting properties among these monolayers may be due to either different hydrolysis efficiencies or variations in surface coverage.

To clarify this question, we also examined the three esterterminated monolayers by ATR-FTIR measurements. Before hydrolysis, the most evident bands observed in the 3200-2700 $\mathrm{cm}^{-1}$ region are the $\mathrm{CH}_{2}$ symmetric stretch $\left(\mathrm{d}^{+}, 2851-2 \mathrm{~cm}^{-1}\right)$ and asymmetric stretch $\left(\mathrm{d}^{-}, 2920-1 \mathrm{~cm}^{-1}\right)$ (Figure 2). The wavenumbers of the $\mathrm{d}^{-}$modes are comparable (Table 1), suggesting that they are of similar quality in terms of packing 
(a)
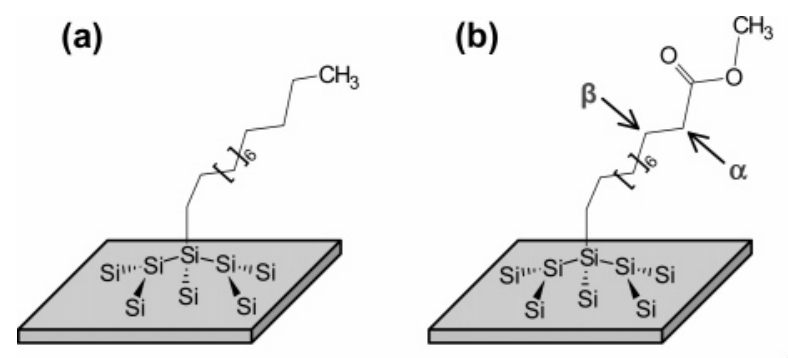

(c)

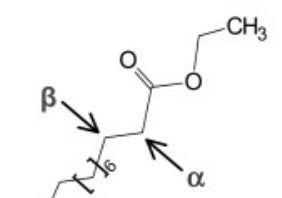

(d)

(d)
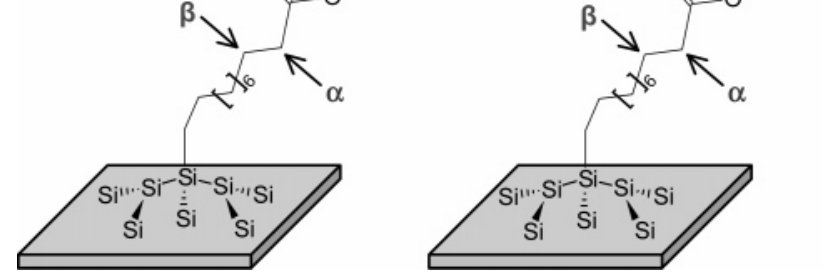

Figure 3. Schematic representations of (a) dodecyl monolayers and ester-terminated monolayers on silicon formed from (b) Me-UD, (c) Et-UD, and (d) Pr-UD.

density and molecular orientation; that is, they are ordered and closely packed monolayers. ${ }^{30}$ However, these bands are broader than those obtained for $n$-alkyl monolayers, which is possibly due to contributions from different types of $\mathrm{CH}_{2}$ groups; for example, the propoxy group has two methylene groups in addition to those linked to the silicon (Figure 3 ).

The carbonyl stretches were observed at $1740-2 \mathrm{~cm}^{-1}$ (Figure 2); upon hydrolysis, this band shifts to $1715 \mathrm{~cm}^{-1}$, accompanied by a shoulder at $1740-2 \mathrm{~cm}^{-1}$. The intensities of the shoulder peaks differ from each other: smallest and least discrete for the Me-UD monolayers, but well-defined at 1740 $\mathrm{cm}^{-1}$ for Pr-UD (insets of Figure 2). The assignment of the bands at $1715 \mathrm{~cm}^{-1}$ is unambiguous: they are attributed to hydrogen-bonded carboxy groups. ${ }^{15,31}$ The shoulder band at $1740 \mathrm{~cm}^{-1}$ may be due to unreacted ester groups or free (nonhydrogen-bonded) carboxy groups (or a combination of both). ${ }^{5 a, 6 b, 15}$ The former interpretation would indicate incomplete hydrolysis, the latter a completely hydrolyzed surface with different surface-bonding properties. The bands at 2983 and $2973 \mathrm{~cm}^{-1}$ for the Et-UD and Pr-UD monolayers are assigned to the $\mathrm{CH}_{3}$ asymmetric stretch $\left(\mathrm{r}^{-}\right)$; their low intensities do not allow further interpretation of the hydrolysis reaction.

While ATR-FTIR and wetting measurements provide some information about the monolayer structure and reactivity, they do not reveal details about the hydrolysis efficiency and potential structural changes. ${ }^{15}$ Therefore, SFG studies of the three esterterminated monolayers before and after hydrolysis were carried out. The spectrum of dodecyl monolayers was also obtained for comparison, while the spectrum of the monolayers prepared from 10-undecylenic acid is included for peak assignment purposes (for which wetting and infrared data have been published previously). ${ }^{32}$ All SFG spectra had signal-to-noise ratios comparable to those reported in the literature. ${ }^{25-27}$

3.1. Peak Assignments and Structure Evaluation. Because SFG vibrational modes must be both IR- and Raman-active, ${ }^{33}$ conventional IR and Raman band assignments are often used as references for the interpretation of SFG spectra. ${ }^{34}$ The most widely characterized alkyl groups, $\mathrm{CH}_{3}$ and $\mathrm{CH}_{2}$, show strong SFG bands in the $2800-3000 \mathrm{~cm}^{-1}$ region. The peaks in this range are generally assigned to $\mathrm{C}-\mathrm{H}$ symmetric $\left(2862-82 \mathrm{~cm}^{-1}\right.$ for $\mathrm{CH}_{3}, 2843-63 \mathrm{~cm}^{-1}$ for $\mathrm{CH}_{2}$ ) and asymmetric stretching (2952-72 $\mathrm{cm}^{-1}$ for $\mathrm{CH}_{3}, 2916-36 \mathrm{~cm}^{-1}$ for $\mathrm{CH}_{2}$ ). These bands
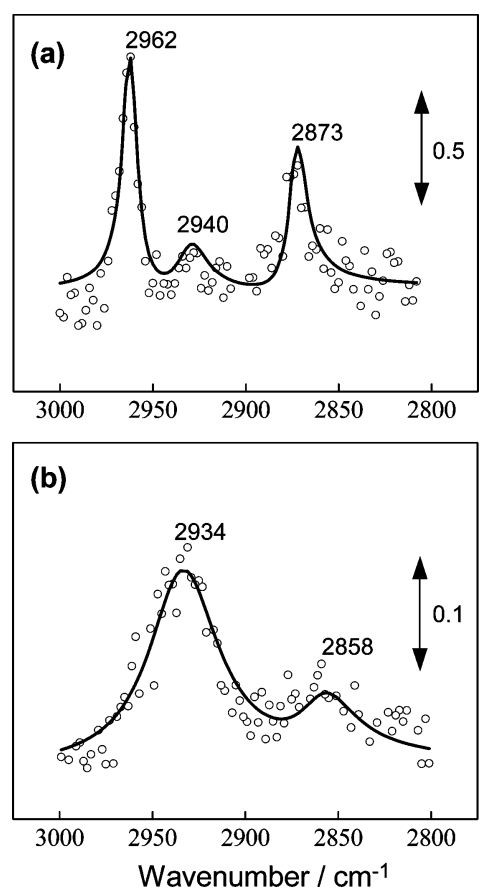

Figure 4. SFG spectra of monolayers prepared from the reaction of (a) 1-dodecene and (b) 10-undecylenic acid with hydrogen-terminated silicon(111). The solid lines are the best fits based on eqs 1 and 2 .

are often accompanied by Fermi resonance (FR) peaks, and there are significant overlaps between $\mathrm{CH}_{3}$ and $\mathrm{CH}_{2}$ stretches. The polarization settings of the laser system add another dimension of difficulty to the interpretation of SFG spectra. All of the above factors lead to ambiguities so that the spectral assignments reported in the literature do not always agree. ${ }^{35}$

The interpretation of the spectrum of 1-dodecyl monolayers on silicon is simple (Figure 4a). The three major bands are due to vibrations of the terminal $\mathrm{CH}_{3}$ groups: $\mathrm{C}-\mathrm{H}$ symmetric stretch $\left(\mathrm{r}^{+}, 2873 \mathrm{~cm}^{-1}\right)$, Fermi resonance between $\mathrm{r}^{+}$and the $\mathrm{C}-\mathrm{H}$ bending overtone (FR, $2940 \mathrm{~cm}^{-1}$ ), and $\mathrm{C}-\mathrm{H}$ asymmetric stretch $\left(\mathrm{r}^{-}, 2962 \mathrm{~cm}^{-1}\right) .{ }^{26,27}$ Although the contributions from $\mathrm{CH}_{2}$ groups were also considered, they are negligible in the optimized fitting results (solid line in Figure 4a). The fact that only terminal $\mathrm{CH}_{3}$ contributes to the SFG signal indicates that essentially all $\mathrm{CH}_{2}$ groups adopt the all-trans configuration, which is consistent with our SFG study of octadecyl monolayers on silicon reported previously. ${ }^{27}$ Ishibashi et al. have observed weak contributions from $\mathrm{CH}_{2}$ stretches (broad bands around 2914 and $2850 \mathrm{~cm}^{-1}$ ), particularly for the $n$-alkyl monolayers that contain odd numbers of carbons. ${ }^{26}$

The peak assignments for ester-terminated monolayers on silicon are challenging because their SFG spectra contain several discernible peaks (Figure 5). At least five bands can be distinguished: $2853-7,2885-90,2902-5,2930$, and 2960-3 $\mathrm{cm}^{-1}$. By analogy to the spectrum of dodecyl monolayers, which is dominated by $\mathrm{CH}_{3}$ stretching modes, the 2885-90 and $2960-3 \mathrm{~cm}^{-1}$ bands can be assigned to $\mathrm{r}^{+}$and $\mathrm{r}^{-}$modes from the terminal methyl groups, respectively. There are few possibilities for the other three bands: $2853-7 \mathrm{~cm}^{-1}$ may be either a $\mathrm{C}-\mathrm{H}$ symmetric stretch of $\mathrm{CH}_{2}\left(\mathrm{~d}^{+}\right)$or $\mathrm{r}^{+}, 2902-5 \mathrm{~cm}^{-1}$ may be either a $\mathrm{C}-\mathrm{H}$ asymmetric $\left(\mathrm{d}_{\omega}{ }^{-}\right)$stretch or Fermi resonance of $\mathrm{CH}_{2}\left(\mathrm{~d}^{-}\right.$or $\left.\mathrm{FR}\right)$, and $2930 \mathrm{~cm}^{-1}$ could be either $\mathrm{d}^{-}$or FR$\left(\mathrm{CH}_{3}\right)$.

Under carefully controlled irradiation conditions, carboxylic acid-terminated monolayers were prepared by the reaction of 10 -undecylenic acid with $\mathrm{H}-\mathrm{Si}^{32,36}$ Their SFG spectrum was 

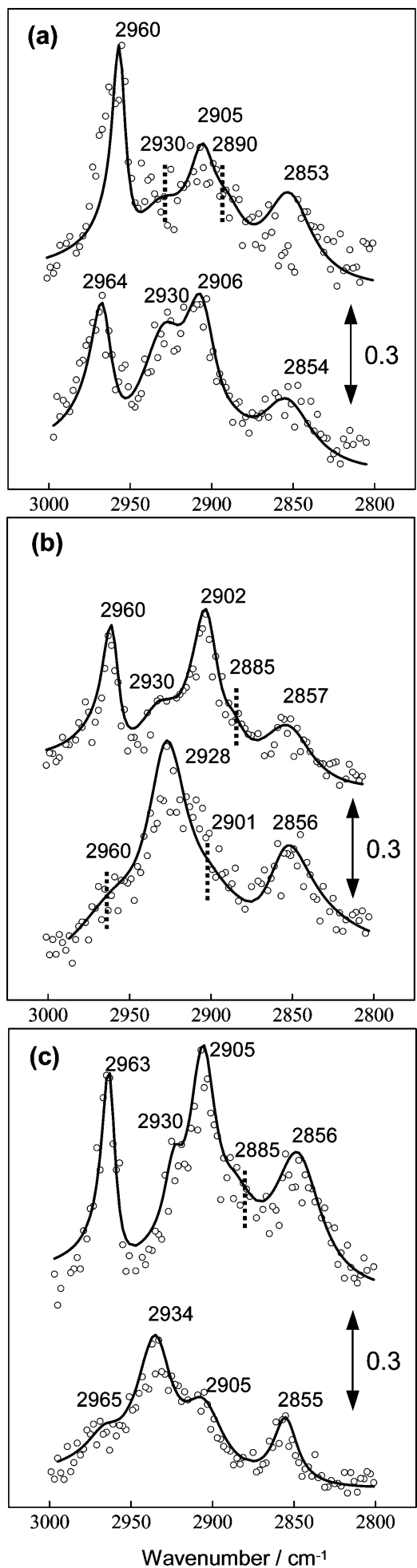

Figure 5. SFG spectra of the ester-terminated monolayers on silicon: (a) Me-UD; (b) Et-UD; (c) Pr-UD. In each panel, the top trace and the bottom trace show the spectrum obtained from the monolayer before and after hydrolysis, respectively. The solid lines are the best fits based on eqs 1 and 2 . The vertical dashed lines indicate the positions of weaker bands.

recorded (Figure 4b) to assist the band assignments for the esterterminated monolayers. Broad peaks were found at 2934 and $2858 \mathrm{~cm}^{-1}$ and were assigned to $\mathrm{d}^{-}$and $\mathrm{d}^{+}$, respectively, since the monolayer formed from 10-undecylenic acid does not contain methyl groups. This agrees well with the ATR-FTIR results reported in the literature. ${ }^{32}$ Accordingly, the bands at $2930 \mathrm{~cm}^{-1}$ and $2853-7 \mathrm{~cm}^{-1}$ of ester-terminated monolayers
TABLE 2: Peak Assignments and Positions $\left(\mathrm{cm}^{-1}\right)$ of the ATR-FTIR Spectra for the Ester-Terminated Monolayers on Silicon (before and after Hydrolysis)

\begin{tabular}{lllll}
\hline & & \multicolumn{3}{c}{ monolayer } \\
\cline { 3 - 5 } & vibrational mode & Me-UD & Et-UD & Pr-UD \\
\hline before hydrolysis & $v_{\mathrm{s}}\left(\mathrm{CH}_{2}\right), \mathrm{d}^{+}$ & 2851 & 2852 & 2851 \\
& $v_{\text {as }}\left(\mathrm{CH}_{2}\right), \mathrm{d}^{-}$ & 2920 & 2920 & 2920 \\
& $v_{\text {as }}\left(\mathrm{CH}_{3}\right), \mathrm{r}^{-}$ & & 2983 & 2972 \\
after hydrolysis & $v(\mathrm{C}=\mathrm{O})$ & 1742 & 1740 & 1740 \\
& $v_{\mathrm{s}}\left(\mathrm{CH}_{2}\right), \mathrm{d}^{+}$ & 2851 & 2850 & 2852 \\
& $v_{\text {as }}\left(\mathrm{CH}_{2}\right), \mathrm{d}^{-}$ & 2921 & 2920 & 2921 \\
& $v_{\text {as }}\left(\mathrm{CH}_{3}\right), \mathrm{r}^{-}$ & & & \\
& $v(\mathrm{C}=\mathrm{O})$ & 1715 & 1715 & 1715 \\
& $v(\mathrm{C}=\mathrm{O})$ & $1740^{a}$ & $1740^{a}$ & $1740^{a}$
\end{tabular}

${ }^{a}$ A shoulder band besides the peak at $1715 \mathrm{~cm}^{-1}$.

TABLE 3: SFG Vibrational Frequencies $\left(\mathrm{cm}^{-1}\right)$ and Their Proposed Resonant Modes for the Ester-Terminated Monolayers

\begin{tabular}{cccc}
\hline & \multicolumn{3}{c}{ monolayer } \\
\cline { 2 - 4 } vibrational mode & Me-UD & Et-UD & Pr-UD \\
\hline$v_{\mathrm{s}}\left(\mathrm{CH}_{2}\right), \mathrm{d}^{+}$ & 2853 & 2857 & 2856 \\
$v_{\text {as }}\left(\mathrm{CH}_{2}\right), \mathrm{d}_{\omega}{ }^{-}$ & 2905 & 2902 & 2905 \\
$v_{\text {as }}\left(\mathrm{CH}_{2}\right), \mathrm{d}^{-}$ & 2930 & 2930 & 2930 \\
$v_{\mathrm{s}}\left(\mathrm{CH}_{3}\right), \mathrm{r}^{+}$ & 2890 & 2885 & 2885 \\
$v_{\text {as }}\left(\mathrm{CH}_{3}\right), \mathrm{r}^{-}$ & 2960 & 2960 & 2963
\end{tabular}

TABLE 4: SFG Vibrational Frequencies $\left(\mathrm{cm}^{-1}\right)$ and Their Proposed Resonant Modes for the Hydrolyzed Ester-Terminated Monolayers

\begin{tabular}{cccc}
\hline & \multicolumn{3}{c}{ monolayer } \\
\cline { 2 - 4 } vibrational mode & Me-UD & Et-UD & Pr-UD \\
\hline$v_{\mathrm{s}}\left(\mathrm{CH}_{2}\right), \mathrm{d}^{+}$ & 2854 & 2856 & 2855 \\
$v_{\text {as }}\left(\mathrm{CH}_{2}\right), \mathrm{d}_{\omega}{ }^{-}$ & 2906 & 2901 & 2905 \\
$v_{\text {as }}\left(\mathrm{CH}_{2}\right), \mathrm{d}^{-}$ & 2930 & 2928 & 2934 \\
$v_{\mathrm{s}}\left(\mathrm{CH}_{3}\right), \mathrm{r}^{+}$ & & & \\
$v_{\text {as }}\left(\mathrm{CH}_{3}\right), \mathrm{r}^{-}$ & 2964 & 2960 & 2965
\end{tabular}

should also be assigned to $\mathrm{d}^{-}$and $\mathrm{d}^{+}$. The band at 2902-5 $\mathrm{cm}^{-1}$ is particularly distinct for the Et-UD and Pr-UD monolayers (Figure $5 b$ and $c$ ), and most difficult to assign. It is possible to attribute this band to $\mathrm{FR}\left(\mathrm{CH}_{2}\right)$, but this would violate the selection rule. ${ }^{34} \mathrm{We}$ are cautiously attributing this band to the asymmetric stretch of $\mathrm{CH}_{2}\left(\mathrm{~d}_{\omega}{ }^{-}\right)$instead, consistent with the assignment of the $\mathrm{C}-\mathrm{H}$ stretching vibrations of octadecylsiloxane on glass by Chow et al. ${ }^{24}$ In our case, there are two different types of $\mathrm{d}^{-}$stretches, 2930 and 2902-5 $\mathrm{cm}^{-1}$; the former can be attributed to trans-gauche defects in the backbone chains, which has been observed by Ishibashi et al. ${ }^{36}$ Since no $2902-5 \mathrm{~cm}^{-1}$ band was observed for $n$-alkyl monolayers on silicon, ${ }^{26,27}$ this peak must be related to $\mathrm{CH}_{2}$ groups adjacent to the carboxyl group (Figure 3). All peak assignments for the ester-terminated monolayers on silicon before and after hydrolysis are summarized in Tables 3 and 4.

The spectrum of dodecyl monolayers on silicon (Figure 4a) is composed of three major bands, $2873 \mathrm{~cm}^{-1}\left(\mathrm{r}^{+}\right), 2940 \mathrm{~cm}^{-1}$ (FR), and $2962 \mathrm{~cm}^{-1}\left(\mathrm{r}^{-}\right)$. The contributions from methylene groups are negligible, suggesting densely packed, all-trans configurations of the alkyl chains. For the ester-terminated monolayers, the methyl-related bands are still strong (Figure 5) but accompanied by significant contributions from $\mathrm{CH}_{2}$ stretches $\left(\mathrm{d}^{+}\right.$and $\left.\mathrm{d}^{-}\right)$. It has been proposed by Ishibashi et al. that gauche defects (particularly the "twisted stems" near the silicon substrate) in the alkyl chains play an important role, since a gauche conformation breaks the local symmetry of the backbone and thereby increases the intensity of the $\mathrm{CH}_{2}$ stretching modes. This indicates that the alkyl chains of ester- 
Me-UD: $r^{-}$

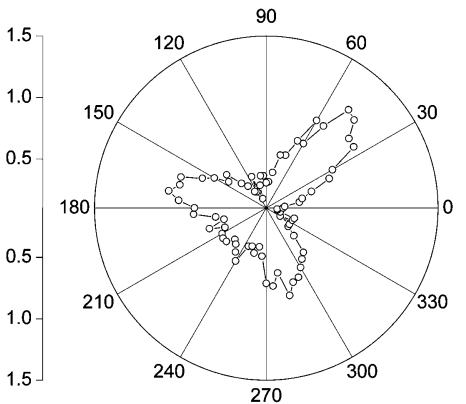

Me-UD: $r$, upon hydrolysis.

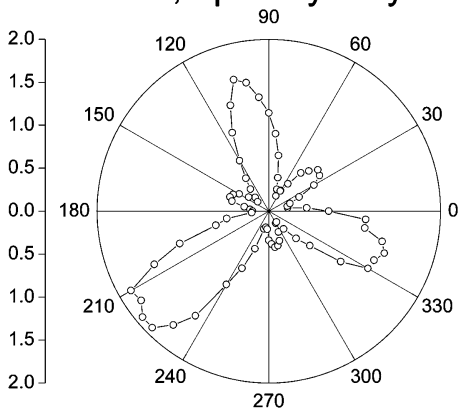

Me-UD: NR

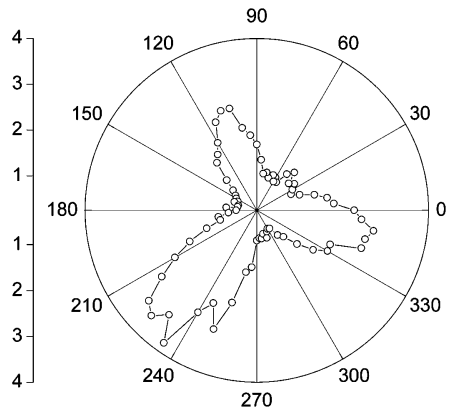

Et-UD: $r$

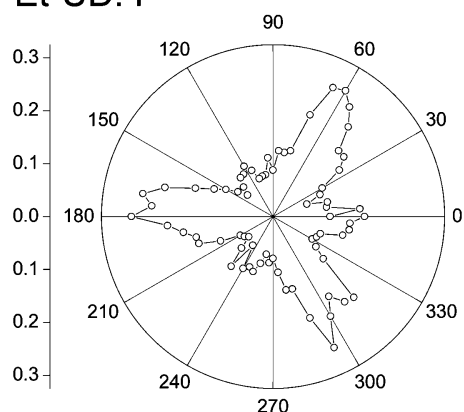

Et-UD: $r$-, upon hydrolysis

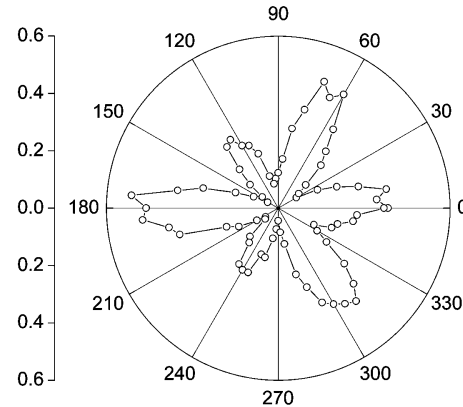

Et-UD: NR

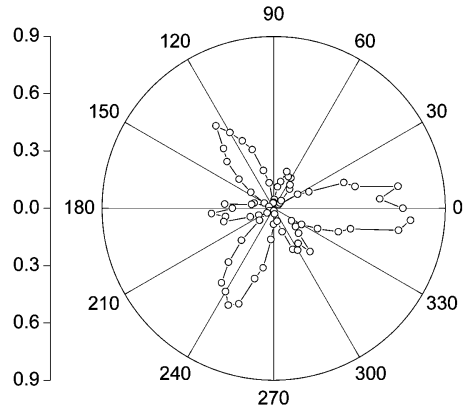

Pr-UD: $r$

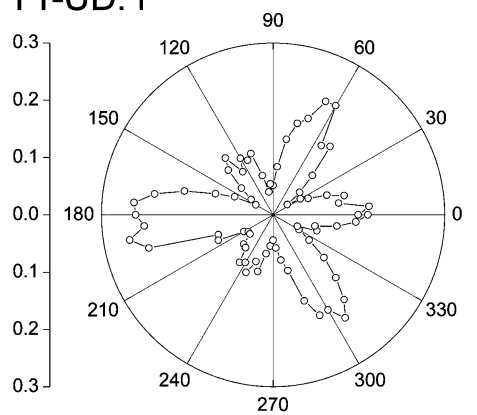

Pr-UD: $r$, upon hydrolysis

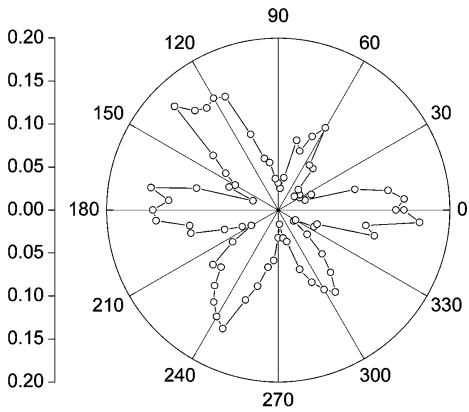

Pr-UD: NR

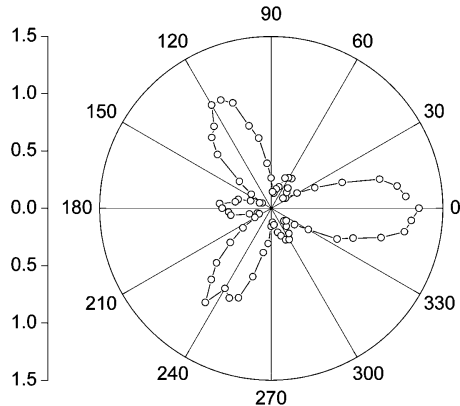

Figure 6. Rotation anisotropy of the SFG intensity of the $\mathrm{r}^{-}$mode (asymmetric $\mathrm{CH}_{3}$ stretch) of the three ester-terminated monolayers on silicon before (top) and after hydrolysis (middle). The nonresonance (NR) signals (bottom) are shown for comparison.

terminated monolayers are less ordered than those of $n$-alkyl monolayers on silicon; that is, the torsional angles of some $\mathrm{C}-\mathrm{C}$ bonds deviate from the ideal value for all-trans conformations. The less prominent $\mathrm{CH}_{3}$ signals also support this hypothesis: structural defects disturb the orientational order of the terminal (methyl) groups (thus weakening their SFG signals). All of these facts show that the ester groups induce distorted structures by prohibiting perfect packing of the alkyl chains, an effect not evident in conventional IR spectra. ${ }^{15}$

Although the number of gauche defects per alkyl chain cannot be calculated quantitatively, the ratio of the $\mathrm{CH}_{3}$ and $\mathrm{CH}_{2}$ signal intensities serves as a reasonable measure of the relative order within the backbone alkyl chains: it increases as a monolayer becomes more oriented. ${ }^{37}$ The calculated $\mathrm{r}^{-} / \mathrm{d}^{-}$ratios $(\sim 2930$ $\mathrm{cm}^{-1} / 2960 \mathrm{~cm}^{-1}$ ) decrease from 2.8 for Me-UD to 1.7 for Et-UD and 1.4 for Pr-UD monolayers, indicating that methyl ester-terminated monolayers (Me-UD) are the most ordered (minimum gauche defects) among the three. This means that the presence of the ester groups plays an important role: the bulkier the ester group, the more disordered the monolayer becomes.

3.2. Hydrolysis Efficiency. The SFG spectra of the hydrolyzed monolayers are shown at the bottom of each panel in Figure 5. The most significant feature of these spectra is that all $\mathrm{CH}_{3}$ bands are still discernible, but their intensities have decreased significantly upon hydrolysis. This confirms that the hydrolysis reaction under acidic conditions is not complete and therefore removes the previous uncertainty about the assignment of the shoulder peak near $1740 \mathrm{~cm}^{-1}$ in the IR spectra (Figure $2)$ : it is definitely and unambiguously due to unreacted ester groups. We believe that steric hindrance is most likely the cause of incomplete acid-catalyzed hydrolysis in these ordered monolayers. The hydrolysis reaction is generally initiated by protonation of the carbonyl oxygen, followed by nucleophilic attack of water to yield a tetrahedral intermediate (i.e., $\mathrm{A}_{\mathrm{AC}} 2$, the most common ester hydrolysis mechanism). Subsequent proton transfer and elimination of alcohol complete the reaction. ${ }^{38 a}$ The alternate alkyl-oxygen cleavage mechanism $\left(\mathrm{A}_{\mathrm{AL}} 2\right)$, which generally requires a sterically hindered carbonyl carbon (which is possible in these ordered monolayers), would also begin with oxygen protonation. ${ }^{38 \mathrm{~b}}$ In either case, the hydrophobic end groups (alkoxy chains) may limit the accessibility of the carbonyl groups to proton/water, and the well-packed monolayer structure may be unfavorable for the generation of bulkier, positively charged intermediates. In contrast, SFG studies by Voges et al. have shown that $\omega$-ester-siloxane monolayers on glass are completely hydrolyzed upon acid treatment (the $\mathrm{CH}_{3}$ bands disappeared completely). ${ }^{23}$ This difference may be attributed to the less ordered, amorphous structure of those monolayers on glass in comparison to the close-packed counterparts formed on crystalline silicon. 
Another equally important feature of the spectra is the increase of the relative intensities of the methylene asymmetric stretching modes $\left(\mathrm{d}^{-}, \sim 2930 \mathrm{~cm}^{-1}\right)$ upon ester hydrolysis. As mentioned above, the $\mathrm{d}^{-}$mode is related to gauche defects in the alkyl chains. The observed intensity increase indicates a significant disruption of the monolayer structure, which may be induced by the bulky intermediate formed during the hydrolysis or by damage to the monolayers upon acid treatment (removal of some alkyl chains). However, the relative intensities of the 2902-5 bands $\left(d_{\omega}{ }^{-}\right)$of the Et-UD and Pr-UD monolayers substantially decreased upon hydrolysis (Figure $5 b$ and $c$ ), indicating that this band is associated with the $\mathrm{CH}_{2}$ groups in the alkoxy chains.

3.3. Rotational Anisotropy. The dependence of the SFG intensity on the azimuthal angle (angle between the incidence plane of the probe beams and the $\left[\begin{array}{lll}1 & \overline{2} & 1\end{array}\right]$ direction of the Si(111) surface) was examined for the three ester-terminated monolayers. A 3-fold pattern of the rotation anisotropy was observed for each of the monolayers at the $\mathrm{r}^{-}$frequency (2960-5 $\mathrm{cm}^{-1}$, the asymmetric $\mathrm{CH}_{3}$ stretching mode) (Figure 6), suggesting the existence of lateral symmetry in these monolayer systems. A similar pattern was observed for a nonresonant frequency (e.g., $2700 \mathrm{~cm}^{-1}$ ) but differed from that of the $\mathrm{r}^{-}$mode by $60^{\circ}$. The rotation anisotropies of these monolayers after hydrolysis are also depicted in Figure 6; they possess significant contributions from both nonresonant and resonant components.

Analogous results have been reported for octadecyl monolayers on $\mathrm{Si}(111)$ in our previous studies. ${ }^{27}$ In that case, the 3 -fold feature was attributed to the orientation of the tilted alkyl chains, based on a comparison with $\mathrm{H}-\mathrm{Si}$ surfaces and alkanethiolate monolayers on gold. ${ }^{27}$ This interpretation, essentially, helps us to gain further insights into the structure and hydrolysis reactivity of the $\omega$-functionalized organic monolayers on silicon. The similarity of the observed rotation anisotropies indicates that the ester-terminated monolayers possess molecular conformations similar to those of octadecyl monolayers; that is, they are reasonably ordered with a high packing density. However, in the presence of ester groups with longer alkoxy chains (e.g., for the Pr-UD monolayers shown in Figure 6, top panels), the nonresonance component becomes more evident; that is, the structure becomes less oriented.

Upon hydrolysis, the rotation anisotropies of these monolayers exhibit both $\mathrm{r}^{-}$and nonresonance contributions (with different relative intensities, as shown in Figure 6, middle panels), indicating the existence of residual ester groups. When the system is dominated by $\mathrm{r}^{-}$, the nonresonance contributions are negligible (Figure 6, top panels). This permits a direct comparison of the hydrolysis efficiencies for the three systems. The Me-UD monolayer upon hydrolysis has the weakest $\mathrm{r}^{-}$band but a strong nonresonant signal; in contrast, the hydrolyzed EtUD and Pr-UD monolayers seem to have signal contributions of comparable intensities at the $\mathrm{r}^{-}$and nonresonance (NR) frequencies. Assuming that the $\mathrm{r}^{-} / \mathrm{NR}$ ratio reflects the relative number of molecules exhibiting the signal for rotation anisotropy at the $\mathrm{r}^{-}$frequency, the equation

$$
\begin{aligned}
& \text { hydrolysis efficiency }= \\
& \qquad \frac{\left(\mathrm{r}^{-} / \mathrm{NR}\right)_{\text {prior to hydrolysis }}-\left(\mathrm{r}^{-} / \mathrm{NR}\right)_{\text {upon hydrolysis }}}{\left(\mathrm{r}^{-} / \mathrm{NR}\right)_{\text {prior to hydrolysis }}}
\end{aligned}
$$

gives the following approximate hydrolysis efficiencies: $97 \pm$ $1 \%$ for Me-UD, $77 \pm 3 \%$ for Et-UD, and $72 \pm 5 \%$ for Pr-UD monolayers. ${ }^{39}$ This is generally consistent with the relative intensity changes for the methyl stretches $\left(\mathrm{r}^{-}\right.$and $\left.\mathrm{r}^{+}\right)$shown in Figure $5,{ }^{40}$ and provides a quantitative measure of the extent of surface reactions on silicon.

\section{Conclusion}

The SFG studies have shown that ester-terminated monolayers on silicon are generally closely packed and ordered, although they have significant gauche defects compared to $n$-alkyl monolayers: the longer the alkoxy chain, the less ordered the monolayer becomes. It has been confirmed that the acidcatalyzed hydrolysis of ester-terminated monolayers is incomplete and its efficiency depends on the bulky ester group. The hydrophobic ends of the alkoxy groups appear to limit the accessibility of the carbonyl carbon to the nucleophile (water), and a well-packed monolayer structure renders the generation of tetrahedral intermediates unfavorable. It has been demonstrated in this work that the SFG technique provides quantitative information on the correlation between molecular structure and surface reactivity in monolayer systems.

Acknowledgment. The authors are grateful to the Natural Science and Engineering Research Council of Canada (NSERC) for financial support. This work was partially supported by a Grant-in-Aid for Scientific Research (KAKENHI) in Priority Area of "Molecular Nano Dynamics" (no. 16072202) from Ministry of Education, Culture, Sports, Science and Technology. H.A. is grateful for the help and advice received from Dr. Ichizo Yagi and Takanobu Yano on the SFG measurements and wishes to thank Dr. Masayuki Okamura and Kanako Takiguchi for their assistance with the sample preparations. H.-Z.Y. would like to thank Dr. Eberhard Kiehlmann for critically reading and editing the manuscript and the Japanese Society for the Promotion of Sciences (JSPS) for a research fellowship.

\section{References and Notes}

(1) For recent reviews, see: (a) Whitesides, G. M. MRS Bull. 2002, 27, 56-65. (b) Carroll, R. L.; Gorman, C. B. Angew. Chem., Int. Ed. 2002, $41,4378-4400$.

(2) (a) Linford, M. R.; Chidsey, C. E. D. J. Am. Chem. Soc. 1993, 115, 12631-12632. (b) Linford, M. R.; Fenter, P.; Eisenberger, P. M.; Chidsey, C. E. D. J. Am. Chem. Soc. 1995, 117, 3145-3155.

(3) (a) Sieval, A. B.; Linke, R.; Zuilhof, H.; Sudhölter, E. J. R. Adv. Mater. 2000, 12, 1457-1460. (b) Wayner, D. D. M.; Wolkow, R. A. J. Chem. Soc., Perkin Trans. 2002, 2, 23-34. (c) Buriak, J. M. Chem. Rev. 2002, 102, 1271-1308.

(4) (a) Wagner, P.; Nock, S.; Spudich, J. A.; Volkmuth, W. D.; Chu, S.; Cicero, R. L.; Wade, C. P.; Linford, M. R.; Chidsey, C. E. D. J. Struct. Biol. 1997, 119, 189-201. (b) Cicero, R. L.; Linford, M. R.; Chidsey, C. E. D. Langmuir 2000, 16, 5688-5695.

(5) (a) Sieval, A. B.; Demirel, A. L.; Nissink, J. W. M.; Linford, M. R.; Van der Maas, J. H.; De Jeu, W. H.; Zuilhof, H.; Sudhölter, E. J. R. Langmuir 1998, 14, 1759-1768. (b) Sieval, A. B.; Vleeming, V.; Zuilhof, H.; Sudhölter, E. J. R. Langmuir 1999, 15, 8288-8291.

(6) (a) Boukherroub, R.; Morin, S.; Bensebaa, F.; Wayner, D. D. M. Langmuir 1999, 15, 3831-3835. (b) Boukherroub, R.; Wayner, D. D. M J. Am. Chem. Soc. 1999, 121, 11513-11515. 10.

(7) Fidélis, A.; Ozanam, F.; Chazalviel, J.-N. Surf. Sci. 2000, 444, L7-

(8) (a) Henry de Villeneuve, C.; Pinson, J.; Bernard, M. C.; Allongue, P. J. Phys. Chem. B 1997, 101, 2415-2420. (b) Allongue, P.; Henry de Villeneuve, C.; Pinson, J.; Ozanam, F.; Chazalviel, J. N.; Wallart, X. Electrochim. Acta 1998, 43, 2791-2798.

(9) (a) Bansal, A.; Li, X.; Lauermann, I.; Lewis, N. S. J. Phys. Chem. $B$ 1996, 118, 7225-7226. (b) Bansal, A.; Lewis, N. S. J. Phys. Chem. B 1998, 102, 1067-1070.

(10) (a) Strother, T.; Cai, W.; Zhao, X.; Hamers, R. J.; Smith, L. M. J. Am. Chem. Soc. 2000, 122, 1205-1209. (b) Lin, Z.; Strother, T.; Cai, W.; Cao, X.; Smith, L. M.; Hamers, R. J. Langmuir 2002, 18, 788-796. (c) Lasseter, T. L.; Clare, B. H.; Abbott, N. L.; Hamers, R. J. J. Am. Chem. Soc. 2004, 126, 10220-10221.

(11) Willner, I.; Katz, E. Angew. Chem., Int. Ed. 2000, 39, 1180-1218. 
(12) Pike, A. R.; Lie, L. H.; Eagling, R. A.; Ryder, L. C.; Patole, S. N.; Connolly, B. A.; Horrocks, B. R.; Houlton, A. Angew. Chem., Int. Ed. 2002, 41, 615-617.

(13) (a) Pike, A. R.; Patole, S. N.; Murray, N. C.; Ilyas, T.; Connolly, B. A.; Horrocks, B. R.; Houlton, A. Adv. Mater. 2003, 15, 254-257. (b) Pike, A. R.; Ryder, L. C.; Horrocks, B. R.; Clegg, W.; Connolly, B. A.; Houlton, A. Chem.-Eur. J. 2005, 11, 344-353.

(14) (a) Wei, F.; Sun, B.; Guo, Y.; Zhao, X. S. Biosens. Bioelectron. 2003, 18, 1157-1163. (b) Wei, F.; Sun, B.; Liao, W.; Ouyang, J.; Zhao, X. S. Biosens. Bioelectron. 2003, 18, 1149-1155.

(15) Liu, Y.-J.; Navasero, N. M.; Yu, H.-Z. Langmuir 2004, 20, 40394050 .

(16) Lee, E. J.; Bitner, T. W.; Ha, J. S.; Shane, M. J.; Sailor, M. J. J. Am. Chem. Soc. 1996, 118, 5375-5382.

(17) (a) Shen, Y. R. The Principle of Nonlinear Optics; John Wiley \& Sons: New York, 1984; pp 67-85. (c) Shen, Y. R. Nature 1989, 337, 519525. (c) Richmond, G. L. Chem. Rev. 2002, 102, 2693-2724.

(18) (a) Zhu, X. D.; Suhr, H.; Shen, Y. R. Phys. Rev. B 1987, 35, 30473050. (b) Hunt, J. H.; Guyot-Sionnest, P.; Shen, Y. R. Chem. Phys. Lett. 1987, 133, 189-192. (c) Guyot-Sionnest, P.; Hunt, J. H.; Shen, Y. R. Phys. Rev. Lett. 1987, 59, 1597-1600. (d) Superfine, R.; Huang, J. Y.; Shen, Y. R. Phys. Rev. Lett. 1991, 66, 1066-1069.

(19) Guyot-Sionnest, P.; Superfine, R.; Hunt, J. H.; Shen, Y. R. Chem. Phys. Lett. 1988, 144, 1-5.

(20) Liu, Y.; Wolf, L. K.; Messmer, M. C. Langmuir 2001, 17, 43294335 .

(21) Ye, S.; Nihonyanagi, S.; Uosaki, K. Phys. Chem. Chem. Phys. 2001, $3,3463-3469$.

(22) Chen. C.; Loch, C. L.; Wang, J.; Chen, Z. J. Phys. Chem. B 2003, 107, 10440-10445.

(23) Voges, A. B.; Al-Abadleh, H. A.; Musorrafiti, M. J.; Bertin P. A.; Nguyen, S. T.; Geiger, F. M. J. Phys. Chem. B 2004, 108, 18675-18682.

(24) Chow, B. C.; Ehler, T. T.; Furtak, T. E. Appl. Phys. B 2002, 74 395-399.

(25) Ye, S.; Saito, T.; Nihonyanagi, S.; Uosaki, K.; Miranda, P. B.; Kim, D.; Shen, Y.-R. Surf. Sci. 2001, 476, 121-128.

(26) Ishibashi, T.; Ara, M.; Tada, H.; Onishi, H. Chem. Phys. Lett. 2003, 367, 376-381.

(27) Nihonyanagi, S.; Miyamoto, D.; Idojiri, S.; Uosaki, K. J. Am. Chem. Soc. 2004, 126, 7034-7040.
(28) Kern, W.; Puotinen, D. A. RCA Rev. 1970, 31, 188-206.

(29) Bain, C. D.; Troughton, E. B.; Tao, Y. T.; Evall, J.; Whitesides, G. M.; Nuzzo, R. G. J. Am. Chem. Soc. 1989, 111, 321-335.

(30) (a) Snyder, R. G.; Strauss, H. L.; Elliger, C. A. J. Phys. Chem. 1982, 86, 5145-5150. (b) MacPhail, R. A.; Straws, H. L.; Snyder, R. G.; Elliger, C. A. J. Phys. Chem. 1984, 88, 334-341.

(31) Cheng, S. S.; Scherson, D. A.; Sukenik, C. N. Langmuir 1995, 11, $1190-1195$ 5018 .

(32) Asanuma, H.; Lopinski, G. P.; Yu, H.-Z. Langmuir 2005, 21, 5013-

(33) Shen, Y. R. Pure Appl. Chem. 2001, 73, 1589-1598.

(34) Colthup, N. B.; Daly, L. H.; Wiberley, S. E. Introduction to Infrared and Raman Spectroscopy, 3rd ed.; Academic Press: San Diego, CA, 1990; Chapter 5.

(35) (a) Lu, R.; Gan, W.; Wu, B.-H.; Chen, H.; Wang, H.-F. J. Phys. Chem. B 2004, 108, 7297-7306. (b) Lu, R.; Gan, W.; Wu, B.-H.; Zhang, Z.; Guo, Y.; Wang, H.-F. J. Phys. Chem. B 2005, 109, 14118-14129.

(36) Voicu, R.; Boukherroub, R.; Bartzoka, V.; Ward, T.; Wojtyk, J. T. C.; Wayner, D. D. M. Langmuir 2004, 20, 11713-11720.

(37) (a) Messmer, M. C.; Conboy, J. C.; Richmond, G. L. J. Am. Chem Soc. 1995, 117, 8039-8040. (b) Conboy, J. C.; Messmer, M. C.; Richmond, G. L. J. Phys. Chem. 1996, 100, 7617-7622.

(38) (a) Lowry, T. H.; Richardson, K. S. Mechanism and Theory in Organic Chemistry; Harper \& Row Publishers: New York, 1976; pp 443449. (b) http://www.rjclarkson.demon.co.uk/candrands/carboxylicacids.htm (accessed Jan 8, 2006).

(39) The signals for rotation anisotropy were taken at the three maximum angles $\left(60,180\right.$, and $300^{\circ}$ for $\mathrm{r}^{-} ; 0,120$, and $240^{\circ}$ for nonresonance); the average of the three values was used for calculating the hydrolysis efficiency, while the differences between them were used to calculate the uncertainty.

(40) In Figure 5a, the residual $\mathrm{CH}_{3}$ stretching signals appear stronger than those in Figure 5b (Et-UD) and c ( $\mathrm{Pr}-\mathrm{UD}$ ), which is partially due the relatively weaker $\mathrm{CH}_{2}$ signals. Absolute peak intensities are also affected by variations of the laser intensity during prolonged operation; therefore, a more reliable estimate of the ester hydrolysis efficiency is obtained from the rotational anisotropy data (Figure 6) that takes the NR contribution as the internal reference. 